\title{
Probabilistic Logic Learning - A Tutorial Abstract ${ }^{\star}$
}

\author{
Luc De Raedt \\ Department of Computer Science, Katholieke Universiteit Leuven, Belgium
}

luc.deraedt@cs.kuleuven.be

Probabilistic logic learning (PLL), sometimes also called statistical relational learning, addresses one of the central questions of artificial intelligence: the integration of probabilistic reasoning with first order logic representations and machine learning. It has received a lot of attention recently and a rich variety of different formalisms and learning techniques have been developed, which are now being applied to applications in network analysis, robotics, bio-informatics, intelligent agents, etc.

This tutorial starts with an introduction to probabilistic representations and machine learning, and then continues with an overview of the state-of-the-art in probabilistic logic learning. We start from classical settings for logic learning (or inductive logic programming) namely learning from entailment, learning from interpretations, and learning from proofs, and show how they can be extended with probabilistic methods. While doing so, we review state-of-the-art probabilistic logic learning approaches and formalisms, such as PRISM, ICL, stochastic logic programs, Bayesian logic programs, CP-Logic and Markov logic.

More detailed introductory overviews on probabilistic logic learning can be found in 122345 .

Keywords: probabilistic logic learning, statistical relational learning, logic and learning, inductive logic programming, logic programming.

\section{References}

1. De Raedt, L.: Logical and Relational Learning. Springer, Heidelberg (2008)

2. De Raedt, L., Frasconi, P., Kersting, K., Muggleton, S. (eds.): Probabilistic Inductive Logic Programming. LNCS (LNAI), vol. 4911, pp. 1-27. Springer, Heidelberg (2008)

3. Getoor, L., Taskar, B. (eds.): An Introduction to Statistical Relational Learning. MIT Press, Cambridge (2007)

4. De Raedt, L., Kersting, K.: Probabilistic logic learning. SIGKDD Explorations 5(1), 31-48 (2003)

5. De Raedt, L., Kersting, K.: Probabilistic inductive logic programming. In: BenDavid, S., Case, J., Maruoka, A. (eds.) ALT 2004. LNCS, vol. 3244, pp. 19-36. Springer, Heidelberg (2004)

\footnotetext{
^ This tutorial is - in part - based on joint work with Kristian Kersting.
} 